\title{
Quantification of Physical Activity in Children and Adolescents: Issues, Challenges and Recommendations
}

\author{
Ajayi-Vincent, O.B \&N. U. Igbokwe \\ Department of Human Kinetics and Health Education, Faculty of Education, Ekiti State University, Ado-Ekiti
}

\begin{abstract}
Physical activity has been understood to be associated with reduction in all-cause mortality obesity, diabetes, hypertension, CVD, certain cancers, osteoporosis and depression. Quantification of physical as energy expenditure is important since childhood for the purpose of preventing future chromic noncommunicable diseases. Accurate and reliable equipment is important and needed to assess the physical activity among the youths. Furthermore, it is important to note that a high quality physical activity in children and adolescent can contribute to achieving helath goals. Meanwhile, many measurement techniques have been questioned hased on the issues and challenges surrounding them when used to measure the amount of physical activity in children and adolescents, most especially those aged below 10 years. Therefore, issues and challenges concerning validation of physical activity measurements in children need to be resolved for the best acurateand reliable measures to be obtained. This paper discusses available methods in the quantification of physical activity, issues and challenges concerning physical activity measurement, and its influences on physical activity assessment in children and adolescents. Techiques that may be used to validate physical activity measurements in children and adolescents are analysed and recommendations proffered.
\end{abstract}

Keywords: Quantification of physical aactivity, energy expenditure, reliabiltiy and validity issues, youths.

\section{Introduction}

Globally nowadays, promoting physical activity (PA) has become a high priority due to very high rates of inactivity-related chronic diseases. Furthermore, there has been a growing interest in assessing and promoting physical among children of which much of this interest emanate from the increases in the prevalence of pediatric obesity (Welket al,, 2000). It has been documented that PA plays an important role in weight control and significant tracking coefficients have been observed for obesity, coronary risk factors and PA and inactivity among youths (Welket al, 2000). It is important to note that a high quality physical activity quantification in children and adolescent can contribute to achieving desirable health goals (Sallis, 2010). Furthermore, measuring a sample of the population can identify groups at high risk and their geographic locations that can be targeted for the purpose of PA intervention. Sallis, 2010).

Regular PA in important to promote physical and mental health and well-being from childhood to adulthood. PA is associated with reductions in all-cause mortality obesity, diabetes, hypertension, CVD, certain cancers, osteoporosis and depression (Mindellet al, 2014). Quantification of PA as energy expenditure is important from childhood for the purpose of preventing chronic non-communicable diseases in adulthood. (Barbosaet al, 2015). Measuring PA in youths poses some problems and these are compounded if it is necessary or desirable to have an assessment of actual energy expenditure as well. (Durnin1990).there are a number of different techniques that have been used to assess PA in a variety of populations. These are self-report questionnaires, activity monitors, pedometers, accelerometers, heart rate monitors, doubly labeled water, and indirect calorimetry and direct observation which have been developed specifically for children. (Welket al, 2000). Each of the measures has specific advantages and disadvantages that must be considered when selecting any of this instrument to measure PA participation in children and youth. (welket al., 2000).

The issues in quantifying PA in children and adolescents are common measurement challenges for any population, but other factors stem from the unique developmental and behavioural aspects of children. Apart from the accurate assessment method it is important to have a perfect understanding of the nature of the individual or individuals being assessed. (Welket al, 2000). Furthermore, understanding the unique movement pattern of the youths is also important and how these patterns influence the various measurements approaches used to assess physical activity.

\section{Movement Pattern And Its Influence On Physical Activity Assessment In Children And Adolescents}

Measuring PA levels in young children offers unique challenges as youths' movement pattern are highly variable, non-structured and generally comprise short and frequent bursts of moderate to vigorous activity (Hands \& Larkin, 2006). Another difficulty is the different rates of maturation and development among same age children. Different measurement tools and protocols have been used to investigate physical activity in 
Quantification of Physical Activity in Children and Adolescents: Issues, Challenges and..

young children but the inconsistency of agreement and valid methods make it difficult to compare the results. (Hands \& Larkin, 2006). Though, some methods such as self-report recall method that are common in the assessment of PA in youth and adults are not appropriate to use for young children because of their inability to accurately recall their activity levels. (Baranowski, 1988). Therefore, the choices of measurement methods suitable for young children that are available to researchers are limited. (Hands \& Larkin, 2006). The most feasible measures for young children are indirect reports by parents or teachers or objective methods such as direct observations, heart rate monitors, or motion sensors such as accelerometers and pedometers (Hands \& Larkin, 2006).

Insights have been provided to the unique nature of children with respect tot heir propensity for PA. Rowland (1998) suggested a biological basis for the differences in activity patterns between children and adults. Rowland noted that children are inherently active mainly because it is physical movement that provides them with the necessary information required by the central nervous system (CNS) for stimulation. For adults, they achieve arousal of the CNS in a variety of non-locomotor activities such as reading, writing, artistic expression, problem solving, and vocational pursuits. (Rowland, 1998). Welket al. (2000) stated that the fact that children of nearly all animal species are more active than adult populations was because all children have an inherent biological need to be active.

Bailey et al. (1995) provides the most detailed insight into the nature of children's activity behaviours through observation using a coding system calibrated against indirect calorimetry, of which the intensity of children's activity every $3 \mathrm{~s}$ over a 12 hour period were recorded. In their study, a system which quantified the duration, intensity and frequency of youth's PA in 15 children aged 6-10 years in Southern California was observed, which gives room for the 'tempo' of PA in children to be characterized, and which they described as the natural variation in rate and intensity of activity events as well as the intervals between activity events. The medium duration of low and moderate intensity activities were $6 \mathrm{~s}$ while the duration for high intensity activities were 3 seconds. Nearly all bouts of vigorous activity (95\%) lasted less than 15 seconds and only $0.1 \%$ of the bouts were longer than a minute. No bout of high intensity activity longer than 10 minutes was recorded. The median duration between high intensity activities was 18 seconds, but ranged between 3 and 21 seconds. Periods of rest were clearly long in proportion to periods of activity, but $95 \%$ of the 'rest' intervals were less than 4 min and 15 seconds.

The findings indicated that children do not remain inactive for extended periods of time. Therefore, Bailey et al. (1995) suggested that short, intermittent bouts of vigorous PA with regular rest periods of longer duration are typical of children which may be necessary for normal growth and development. This findings and suggestions were consistent with national Association for sport and Physical Education's (NASPE) guidelines on PA for children (Council for Physical Education for Children, 1998) which focused on the volume of activity and highlight that discontinuous activity may characterize youth's behavior more than continuous activity. This document also highlights a number of other cognitive and behavioural differences between adults and children that should be considered when studying or promoting PA in children which also have implications for the assessment of PA (Table 1).

The fact that children have different patterns of activity, either discontinuous of continuous, demands that different intervals of assessment and / or outcome measures be used to assess their levels of activity. The less developed cognitive skills of children regarded as concrete thinking may result in a lesser ability to effectively use self-report questionnaires. Biological differences in metabolism and biomechanical differences in efficiency and economy require different assumptions for measurement techniques that aim to estimate energy expended in PA (Welk et al. 2000).

Table 1.Characteristics that differentiate children from adults in Physical Activity

\begin{tabular}{|c|c|c|}
\hline $\mathrm{T}$ y p e s & $\begin{array}{llllllllllllll}\mathrm{C} & \mathrm{h} & \mathrm{a} & \mathrm{r} & \mathrm{a} & \mathrm{c} & \mathrm{t} & \mathrm{e} & \mathrm{r} & \mathrm{i} & \mathrm{s} & \mathrm{t} & \mathrm{i} & \mathrm{c}\end{array}$ & $\begin{array}{llllll}\mathrm{I} & \mathrm{m} & \mathrm{p} & \mathrm{l} & \mathrm{i} & \mathrm{c}\end{array}$ \\
\hline \multirow[t]{3}{*}{ Biological } & Need for high level of central nervous system arousal & High volume of physical activity is typical \\
\hline & & Low tolerance for total inactivity \\
\hline & & Spontaneous activity is common \\
\hline \multirow[t]{4}{*}{ Cognitive functioning } & More concrete (less abstract) thought process & Relatively short attention span on any given task \\
\hline & & Less interest in continuous activity \\
\hline & & Failure to see long term benefits of activity (e.g health benefits) \\
\hline & L e s s d e v e lo p e d c o g n i t i o $n$ & 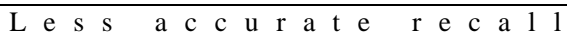 \\
\hline
\end{tabular}


Quantification of Physical Activity in Children and Adolescents: Issues, Challenges and..

\begin{tabular}{|c|c|c|}
\hline & & Inability to accurately estimate time \\
\hline \multirow[t]{2}{*}{ Physiological } & Limited tolerance for vigorous physical activity & Activity typically intermittent in nature \\
\hline & Weak relationship between fitness an physical activity & 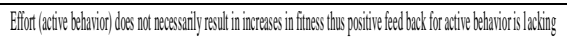 \\
\hline \multirow[t]{2}{*}{ Biomechanical } & Poorer economy and efficiency of movement & Quicker onset of fatigue, and need for frequent rest \\
\hline & & Less interest in continuous activity \\
\hline \multirow[t]{2}{*}{ Psychological } & More a vailable free ti me & More time to try new activities \\
\hline & Natural curiosity and desire for pursuing new task & Interest in exploring new activities \\
\hline
\end{tabular}

NASPE Guidelines on Physical Activity for Children. Adapted from (Welk et al., 2000)

\section{Physical Activity Quantification Techniques In Children And Adolescents}

PA is seen as a broadly used term, for which its diverse nature makes it very difficult to characterize and quantify (Goran, 1998(. PA can be regarded as any physical movement which comes as a result of skeletal muscle contraction (Caspersenet al., 1985). Although, PA is often being measured in terms of caloric cost, this may not be suitable, because the benefits and health effect of PA using a high-energy expenditure (e.g running) versus a low energy expenditure (e.g strength training) may not be related to the caloric cost of the PA (Groan, 1998). Therefore, in quantifying PA, al aspects which includes the type and purpose of PA an intensity, fitness and well-being should be considered (Goran, 1998).

Another additional difficulty with the development of rigorous techniques for measuring PA is the lack of an ideal standard with which the data can be validated any given technique (Goran, 1998). There are various methods that are available for assessing PA in children. These include questionnaires, accelerometry or pedometry, double labeled water for assessment of free-living physical activity - related energy expenditure, and heart rate monitoring. It will also be good to note that the specific operational definition of PA depends on how it is measured and scored. (Welket al., 2000). The variables of frequency, intensity and duration are commonly used to characterize activity patterns coupled with interest in energy expenditure which is a summary variable that incorporates all other indications. (Welket al., 1995).

There are numbers of different techniques that can be used to obtain a similar out come measure, however, the simplicity and accuracy of measuring these different components differs based on each of the instruments used. The varieties of the techniques that have been developed could be divided into three main categories (Sirard\& Pate, 2001):

1. Primary measures: e.g direct observation, doubly labeled water, and indirect calorimeter

2. Secondary measures: e.g heart rate monitors, pedometers, motion sensors and accelerometers.

3. Subjective measures: e.g self report, interview, proxy report and diary.

\subsection{Primary measures}

\subsubsection{Direct observation}

Direct observation is the most practical and appropriate criterion measure of PA and patterns of activity (Sirard\& Pate, 2001). The assessment of PA has been performed through direct observation in a variety of realistic situations, but the protocol of assessment was varied (Trost, 2007). In the past, it involves observing a child either at home or school for a long period of time and recording data into a handheld laptop computer or coding form for quick rating (Kohl et el., 2000, Trost, 2007). Activity category ratings are usually recorded on a momentary time-sampling basis at time intervals ranging from 5 seconds to 1 minute. Relative to other methods, direct observation has a number of important advantages. Observational procedures are flexible and permit researchers to not only quantify PA but also record factors related to PA behavior cues, environmental conditions, the presence of significant others, and availability of toys and equipment (Trost, 2007).

Direct observation also has an ability the duration, intensity, and frequency of specific activity events (Bailey et al 1995). Despite its flexibility, observation technique to assess PA can be used as either a process or put come measure. Some of the limitations of direct observation of PA despite its usefulness to researchers, are the tedious data-coding requirements and the fact that it is highly labour intensive and expensive (Trost, 2007). Furthermore, it is not practical for large population studies of PA because it is expensive, but can be useful for small studies (Kohl et al., 2000). It is also useful for studies in young children who have not developed the cognitive ability to recall comprehensive data, and can be difficult to implement in a large area, except the participants are restrained in a defined space such as school playground, gymnasium of home (Kohl et al, 2000). Participants' reaction to observers is also a distraction, but can be reduced by doing repeated observations. 


\subsubsection{Indirect calorimetry and Doubly-labeled water}

Indirect calorimetry and doubly-labeled water techniques are considered to be the gold standard assessments for laboratory and field-based studies on PA (Trost, 2007); Welk et al., 2000). In controlled conditions, indirect calorimetry can be used to divide energy expenditure associated with (1) resting metabolic rate (RMR) (2) thermic effect of food (TEF), and (3) thermic effect of exercise (TEE) (Welk et al., 2000). These variables can be used to understand the associations between energy expenditure and body weight control in an individual (Welk et al., 2000). The condition requiredin using indirect calorimeter can possibly be achieved in adults, but might be more difficult in children and adolescents. This may be due to the basal metabolic rate which includes assessment that can be performed after 10-12 hour of fasting in an unaroused state in the laboratory once the participant is awake (Goran, 1998). One of the main disadvantages of using indirect calorimetry to measure resting metabolic rate is that measurements can be performed over only a very short time (30 minutes) (Goran, 1998). Although assessments of more than 24 hours has been achieved in children of 4 years of age so that the activity and food intake can be monitored and controlled, but the main problem here is that chamber environment is not habitual because participants' movement and PA may be restricted. (Goran, 1998).

In the case of doubly-labeled water assessments, a direct measure of carbon-dioxide production, of which, if combined with the proportion of food diet, could yield a highly accurate estimates of energy expenditure (Welk et al., 2000). Doubly-labeled water has several advantages for assessing total daily expenditure in children. It is a non-invasive device that can measure activity over 1-2week periods and may not inhibit the normal PA patterns (Welk et al, 2000; Goran, 1998; Trost, 2007). The main constraints of doubly labeled water in assessing PA in children and adolescents are related to cost. In other words, it is too expensive. Also, the attainment of the stable isotope of water is seen as very difficult, coupled with its usefulness to examining pattern of PA or for separating energy expenditure linked with PA (Welk at al, 2000; Trost, 2007). Additionally, doubly labeled water may be the most accurate method for assessing total energy expenditure, but cannot be relied on, when it comes to producing information on duration and frequency of the PA events (Bailey et al, 1995).

\subsection{Secondary Measure}

\subsubsection{Heart rate monitors}

Heart rate monitor is an expensive device which has a multiple day storage capacity for minute-byminute quantification of PA. Heart rates monitoring technique has been seen as a more feasible method for assessing PA in children and adolescents (Trost, 2007). It was the first widely used objective measure of PA in children, but not a direct measure of PA (Rowland \&Eston, 2007). Therefore, it provides a sign of the relative stress placed upon the cardiopulmonary system by PA (Armstrong, 1998). Because of the direct association between hear rate and expended energy during steady state exercise, it remain a good method of assessing PA (Trost, 2007). On the other hand, there are many factors which can influence (disadvantages) this method. Factors such as age, body size, proportion of muscle mass used, emotional stress and cardio respiratory fitness has been seen as influencer to the heart rate (HR)- $\mathrm{VO}_{2}$ relationship (Trost, 2007; Armstrong \&Welsman, 2006). Furthermore, heart rate monitoring may mask the sporadic activity patterns of children due to the lagging behind of heart rate response during movement and tends to remain elevated after the cessation of movement (Trost, 2007; Rowland \&Eston, 2007).

\subsubsection{Pedometers}

Pedometers have been shown to be a useful tool for quantifying PA in children. A pedometer is a costeffective alternative to accelerometers to measure PA (Trost, 2007). Its main advantages are low cost, compared with other devices and the simplicity of the data produced through the number of steps taken and can be useful for large population surveys (Mindell et al, 2014. Pedometers have also been understood to have the same constraint as accelerometers. The constraint is that they are insensitive to some forms of movements (Trost, 2007). Additionally, this device is incapable to record the magnitude of the movement detected such as walking, running or jumping (Mindell et al, 2014; Welk et al, 2000; Rowland \&Eston, 2007). Furthermore, despite the advancement in producing sophisticated pedometers which can now offer data recording and downloading features, most pedometers, especially those used in schools and health promotion programmes do not possess real-time storage of data and downloading capabilities (Trost, 2007). Therefore, most pedometers can only provide an estimate of the relative volume of activity performed over a specified period such as a day or week, assuming that most of the activity performed involves ambulatory movement such as walking (Trost, 2001). In other word, many viable available pedometers provide the researchers with raw estimates of energy expenditure of which its algorithms were developed from adult studies which may not be valid in children and adolescents (Trost, 2007). 
Quantification of Physical Activity in Children and Adolescents: Issues, Challenges and..

\subsubsection{Accelerators}

Accelerators were introduced in the last three and half decades and have been used to assess PA and to validate self-reported PA (Mindell et al, 2014). Accelerometry-based motion sensors is one of the most regularly used methods for quantifying PA in free-living individuals (Trost, 2001). An accelerometer is an objective device similar to a pedometer which can assess movement directly (Rowland \&Eston, 2007). Accelerometers can measure movement in one, two or three dimensions of uni-, bi- or tria-axial measurements of which tri-axial accelerometers may provide a more valid estimate of children's PA than uni-axial accelerometers (Mindell et al, 2014; Rowland \&Eston, 2007). This makes it a unique device when assessing the association between health and activity (Mindell at al, 2014).

Furthermore, accelerometers are built with a time-sampling capability that can allow the quantification of the temporal pattern and intensity of activity as well as total accumulated activity (Rowland \&Eston, 2007). It also provides quantitative information regarding the vertical accelerations of the trunk or other body segments at user-specified time intervals, in other words, it can be used to evaluate the frequent, intensity and duration of PA over specified time intervals such as days or weeks (Trost, 2007). Their portability, healthy design characteristics and relatively modest cost make them good-looking to researchers who specialized in the area of assessing children and adolescents PA behavior (Trost, 2007).

Some of the disadvantages of accelerometers is that they are not able to account for the increased cost linked to walking up stairs or a slope and do not accurately assess activities that have to do with cycling, lifting or carrying (Trost, 2001; Trost, 2007; Welk et al, 2000). This was due to an assumption that the contribution of these activities to the overall PA is minor. Consequently, accelerometers may undervalue total or PA energy expenditure (Trost, 2007). Furthermore, Rowland and Eston (2007) stated that there is a lack of standardization regarding how accelerometers are used, which outcome measures are used and how the output is interpreted which limits comparability between studies and the accumulation of knowledge relating to children's activity.

\subsubsection{Combined heart rate monitoring and Accelerometry}

The combined heart rate monitoring and accelerometry defined another advancement to improve heart rate-derived estimates of free-living energy expenditure precisions. Several investigators have used a combination of heart rate monitoring and Accelerometry (Treuth et al, 1998; Eston et al, 1998) and concluded that the combination heart rate monitoring and Accelerometry provides an acceptable method for estimating energy expenditure, not only for groups of children but also for individuals (Trost, 2007, Mindell et al, 2014). This approach, developed by researchers at the University of Cambridge in the United Kingdom, has resulted in the release of the Actiheart. The actiheart device monitor weighs approximately 8grams and is attached directly to the chest with 2 standard electrocardiogram (ECG) electrodes. The Actiheart is able to measure acceleration, heart rate, heart rate variability and ECG magnitude in time intervals or epochs of 5, 30 and 60 seconds (Trost, 2007). This combined sensor technology can be quite worrying for the participant because, it may sometimes require removal of hair from men's chest and a risk that gains in accuracy may be offset by losses in participant adherence (Mindell et al, 2014). Furthermore, this device is also expensive and cannot be afforded by many researchers in the field.

\subsection{Subjective measures}

\subsubsection{Self-Report Measure}

Self-report measure provides an easy way to estimate activity patterns in youths most especially on a large population (Welk et al, 2000; Goran, 1998). Various self-report techniques have been used to assess PA in children and adolescents. These include self-administered recalls, interviewer administered recalls, diaries and proxy reports which can be completed by parents or teachers (Trost, 2007). Based on the purpose of the study, self-report measure differs considerably in the specificity with which type, duration, frequency and intensity are assessed. The recall time period frame ranges from as little as one day to as much as one year.

In terms of self-report recall, children younger than 10years may not have the capacity to report their activities accurately (Trost et al, 2007; Baranowski et al, 1984), because it might be difficult for them to differentiate between sedentary activities such as playing computer games and active pursuits such outdoor games and household chores (Trost et al, 2000). Therefore, investigators must be extreme cautious when attempting to use self-report instruments in children aged 10years or younger (Trost, 2007). Parents also cannot report school-based activity and informal play is particularly difficult to assess (Mindell et al, 2014).

In addition, the generally low-validity coefficients observed for self-report instruments in younger children support the idea that objective measures of PA such as accelerometers may be more suitable in primary school-aged children (Trost, 2007). For large samples of children, however, objective measures may be too expensive and logistically too difficult to administer effectively (Trost, 2007). Hence, population-level intervention and surveillance studies involving children aged 10years or younger may have to rely on parental reports of children's PA participation (Trost, 2007). The most often cited advantages of self-report measures are: 
1. Ease of administration

2. The ability to characterize activity historically, and low cost.

3. The ability to record activity type and the context in which PA is performed.

Therefore, self-reports are commonly used in epidemiological research and surveillance studies where objective measurement techniques are often not practical (Trost, 2007).

\section{Conclusion}

In conclusion, the issues and challenges of PA quantification in children and adolescents were reviewed based on characteristics such as cost or affordability, ease of administration or compliance, probability for reactivity and viability in large studies. The assessment instrument to be used largely depends on the scope and aims of the study. This paper reveals that secondary or objective measures such as Accelerometry, heart rate monitoring, pedometry and combined heart rate monitoring and accelerometers may be more efficient compared to others, most especially among younger aged children.

This paper also indicates that each technique has its own advantages and disadvantages which need to be carefully considered when selecting PA measurement equipment for use among children and adolescents, coupled with the understanding that not one single technique can be described as ideal. Furthermore, based on the review in this paper, multiple techniques such as a combination of heart rate monitoring and accelerometers could provide a great deal of efficacy and accuracy. This may suggest that in the quantification of PA among the youths, combination of two or more devices may be more efficient and could reduce the levels of limitations observed when a single device is used.

\section{References}

[1]. Armstrong, N. (1998). Young people's physical activity patterns as assessed by heart rate. Journal of Sports Sciences. 16, S9-S16.

[2]. Armstrong, N. \&Welsman, J. R. (2006). The physical activity patterns of European youth with reference to methods of assessment. Sports Medicine 36, 1067-1086.

[3]. Barawonski, T. (1998). Validity and reliability of self-report measures of physical activity: an information-processing perspective. Research Quarterly for Exercise and Sports, 59(4),314-327.

[4]. Barawonski, T., Dworkin, R. J., Cieslik, C. J., Hooks, P., Clearman, D. R., Ray, L., Dunn, J. K., \& Nader, P. R. (1984). Reliability and validity of self-report of aerobic activity: Family Health Project. Research Quarterly for Exercise and Sports, 55(4),309-317.

[5]. Barbosa, N., Sanchez, C. E., Patino, E., Lozano, B., Thalabard, J., Le Boec, S., \&Rieu, M. (2015). Quantification of physical activity using the QAPACE questionnaire: A two stage cluster sample design survey of children and adolescents attending urban school. The Journal of Sports Medicine and physical fitness.

[6]. Durnin, J.V.G.A. 91990). Methods to assess physical activity and the energy expended for it by infants and children. Activity, energy expenditure and energy requirements of infants and children, 45-55.

[7]. Eston, R.G., Rowlands, A..V., \&Ingledew, D.K. 91998). Validity of heart rate, pedometry, and Accelerometry for predicting the energy cost of children's activities. Journal of applied physiology, 84(1), 362-371

[8]. Goran, M.I. (1998). Measurement issues related to studies of childhood obesity: assessment of body composition, body fat distribution, physical activity, and food intake. Pediatrics, 101(Supplement 2), 505-518.

[9]. Hands, B., \& Larkin, D. (2006. Physical activity measurement methods for your children: A comparative study. Measurement in Physical Education and Exercise Science. 10(3). 203-214.

[10]. Kohl, H. W., Fulton, J.E. \&Caspensen C.J. (2000). Assessment of physical activity among children and adolescents: a review and synthesis. Preventive Medicine, 31(2), S54-S76.

[11]. Mindell, J.S. Coombs, N., \&Stamatakis, E. (2014). Measuring physical activity in children and adolescents for dietary surveys: practicalities, problems and pitfalls. Proceedings of the Nutrition Society, 73(02), 218-225.

[12]. Roland, A.V. \&Eston, R. G. (2007). The measurement and interpretation of children's physical activity. Journal of Sports Science and Medicine, 6, 270-276.

[13]. Sallis, J.F. (2010). Measuring physical activity: practical approaches for program evaluation in Native American communities. Journal of public health management and practice: JPHMP, 16(5), 404.

[14]. Sirad, J. R. \& Pate, R.R. (2001). Physical activity assessment in children and adolescents, Sports medicine, 31(6, 439-454.

[15]. Treuth, M. S. Adolph A. L. \& Butte N. F. 91998). Energy expenditure in children predicted from heart rate and activity against respiration calorimetry. American Journal of Physiology- Endocrinology and Metabolism, 275(1), E12-E18.

[16]. Trost, S.G. (2001) Objective measurement of physical activity in youth: current issues, future directions. Exercise and Sport Sciences Reviews. 29(1), 32-36

[17]. Trost, S.G. (2007). State of the art reviews: measurement of physical activity in children and adolescents. American Journal of Lifestyle Medicine. I(4), 299-314.

[18]. Welk, G. J. , Corbin, C.B. \& Dale, D. (2000). Measurement issues in the assessment of physical activity in children. Research quarterly for exercise and sport, 71(sup2), 59-73 